\title{
Initial Experience of Laparoscopic Pectopexy for Apical Prolapse in South Korea
}

\author{
Eun Hee Yu, Ha Eun Jung, Hye Kyung Noh, Jong Kil Joo \\ Department of Obstetrics and Gynecology, Pusan National University School of Medicine, Pusan National University Hospital Medical \\ Research Institute, Busan, Korea
}

\begin{abstract}
Pelvic organ prolapse (POP) is the herniation or bulging of the pelvic organs to or beyond the vaginal introitus. POP is a common condition affecting more than half of parous women. We recently performed POP surgery and repair in 37 patients using laparoscopic pectopexy, a new technique for apical prolapse surgery, in women with advanced POP. The surgery was performed by a single surgeon at a single institute. The methods of operation were divided into total laparoscopic hysterectomy with pectopexy, supracervical hysterectomy with pectopexy or pectopexy alone, with additional anterior or posterior colporrhaphy selectively performed. All patients were analyzed in terms of age, body mass index, parity, estimated blood loss (EBL), operation time, intraoperative complications, and postoperative complications. The patients were followed up for at least 6 months after surgery, and the short-term clinical outcomes were analyzed. All operations were performed successfully and without severe intraoperative or postoperative complications. The mean EBL was 84 $\mathrm{mL}$, and the mean operation time was 121 minutes. The operation satisfaction rates were high in most patients. All patients had no recurrence of apical prolapse, de novo urgency, frequency, incontinence, de novo constipation, or ileus. In our experience, laparoscopic pectopexy is a feasible method for apical prolapse repair as it does not have severe intraoperative or postoperative complications and de novo gastrointestinal complications. Considerable follow-up period for possible postoperative events is warranted.
\end{abstract}

Key Words: Colporrhaphy, Pectopexy, Pelvic organ prolapse

\section{INTRODUCTION}

The prevalence of pelvic organ prolapse (POP) based on symptoms is $3 \%-6 \%$ and rises up to $50 \%$ when based upon vaginal examination [1]. This discrepancy was due to the absence of POP symptoms in most women [2]. In Korean women, the prevalence of POP was $180 \pm 4$ per 100,000 population, and the number of women requiring surgery were $89 \pm 1$ per 100,000 population for women over 50 years of age [3].

For POP, effective support of the specific prolapsed point of pelvic organ is an essential element of any surgical treatment for advanced POP. Various approaches have been introduced for decades, but it is still challenging to treat for advanced POP accompanied with high recurrence rate. However, since the beginning of mesh implant use in POP surgery, reoperation rate has significantly decreased [4].

An abdominal approach with prosthetic sacrocolpopexy is considered the gold standard for the treatment of apical defects $[5,6]$. Nevertheless, this procedure is still related with some complications such as defecation disorders, ileus and small bowel obstruction [7-9]. In 2010, new type of prolapse surgery, pectopexy, was introduced by Banerjee and Noé [10].

We recently performed prolapse surgery in 37 patients using pectopexy in women with advanced POP and would like to report the initial but significant shortterm experience in this paper. 


\section{CASE REPORT}

A total of 37 women who underwent laparoscopic pectopexy between March 2018 and August 2019 at the Pusan National University Hospital, Busan, Korea, were included. All patients were pelvic organ prolapse quantification system (POP-Q) II or above and presented with symptoms related to apical prolapse, such as visualization and/or sensation of a bulge/protrusion from vagina, discomforts related to sexual intercourse or other related urinary symptoms. All patients were without previous operations for POP correction and pelvic inflammatory disease.

According to patients' will, three kinds of operation were conducted; total laparoscopic hysterectomy with pectopexy, supracervical hysterectomy with pectopexy and pectopexy alone. Anterior and/or posterior colporrhaphy was performed selectively depending on findings of pelvic examination.

A $10 \mathrm{~mm}$ trocar was inserted from the umbilicus, and pneumoperitoneum was generated. Two additional 5 $\mathrm{mm}$ ports were inserted; left and right from $2 \mathrm{~cm}$ medial and superior to the anterior superior iliac crests. After entering intraperitoneal cavity, the round ligament and external iliac vein were visualized. Soft tissue between these structures was pushed downward with blunt dissection, so an approximately $4-5 \mathrm{~cm}$ segment of the right iliopectineal ligament (Cooper ligament) could be exposed. The same step was then repeated on the contralateral side. The peritoneal layers on both sides were opened toward the vaginal apex. In patients performed with total hysterectomy, the anterior and posterior peritoneum of the vaginal stump was dissected for the mesh fixation. In case of supracervical hysterectomy, the peritoneum around cervix was dissected. In other patients wanted to preserve uterus, the anterior peritoneum of the uterus was dissected, and the lower anterior segment of the uterus was prepared. After completion of dissections, $3 \mathrm{~cm} \times 15 \mathrm{~cm}$ mesh was inserted into the pelvic cavity. The center of the mesh in the tension-free position was fixed to the anterior uterine wall, vaginal stump, or cervix with 2 or 3 stitches via the nonabsorbable sutures. The ends of the mesh were cut according to the size of the patient pelvis and the modified ends were fixed to both iliopectineal ligament on the same ways. Finally, the peritoneum above the mesh was closed completely with an absorbable suture material to avoid intra-pelvic adhesions.

Over the study period, 37 patients underwent laparo- scopic pectopexy procedures. The half of patients, 18 patients, underwent laparoscopic supracervical hysterectomy, petopexy and anterior-posterior colporrhaphy. All operations were performed without intraoperative and resulted no postoperative complications.

Data of operation and short-term follow-up were presented in Tables 1 and 2. In two of the eight cases in the vault prolapse, the operation time was longer than other cases due to adhesion of multiple operative history. Of the total 37 patients, 30 patients underwent anterior colporrhaphy, posterior colporrhaphy, or anterior-posterior colporrhaphy, which required additional operation time and resulted in extended total operation time.

The operation satisfaction was assessed based on the discomfort that patients complained about every outpatient visit and the satisfaction rates were high in most patients, but some patients had complaints about recurrent urinary symptoms (Table 2 ). The mean postoperative follow-up duration was 14.2 months.

Table 1. Patients' characteristics and results of operations

\begin{tabular}{lc}
\hline \multicolumn{1}{c}{ Characteristic } & Value \\
\hline Number of patients & 37 \\
Mean age (y) & $66(45-83)$ \\
Mean body mass index $\left(\mathrm{kg} / \mathrm{m}^{2}\right)$ & $25(16-32)$ \\
Mean number of vaginal delivery & $3(0-6)$ \\
Operation method & \\
Laparoscopic total hysterectomy & $4(10.8)$ \\
Laparoscopic supracervical hysterectomy & $21(56.8)$ \\
Pectopexy (uterus preserving) & $5(13.5)$ \\
Vault pectopexy & $7(18.9)$ \\
Colporraphy & $30(81.1)$ \\
Anterior colporrhaphy & $2(5.4)$ \\
Posterior colporrhaphy & $3(8.1)$ \\
Anterior-Posterior colporrhapy & $25(67.6)$ \\
Mean estimated blood loss (mL) & $84(25-300)$ \\
Operation time (min) & $121(85-205)$ \\
Laparoscopic total hysterectomy & $121(118-132)$ \\
Laparoscopic supracervical hysterectomy & $123(90-155)$ \\
Pectopexy (uterus preserving) & $93(85-103)$ \\
Vault pectopexy & $128(90-205)$ \\
Intraoperative complications & 0 \\
Postoperative complications & 0 \\
\hline
\end{tabular}

Data are presented as number only, mean (range), or number (\%). 
Table 2. Results of short-term follow-up

\begin{tabular}{lc}
\hline \multicolumn{1}{c}{ Parameters } & Value \\
\hline Number of patients & 37 \\
Mean postoperative follow-up duration (mo) & $14.2(7-31)$ \\
Satisfied with the surgery & $33(89.2)$ \\
Recurrence of pelvic organ prolapse & 0 \\
Recurrence urinary symptoms & $6(16.2)$ \\
Urgency & 0 \\
Frequency & $4(10.8)$ \\
Incontinence & $2(5.4)$ \\
De novo urinary symptoms & 0 \\
De novo constipation & 0 \\
De novo ileus & 0 \\
\hline
\end{tabular}

Data are presented as number only, mean (range), or number (\%).

\section{DISCUSSION}

In 2010, Banerjee and Noé [10] introduced pectopexy as a new technique of prolapse surgery for obese patients. They suggested that despite the safe consent for sacropexy as adequate approach, the laparoscopic approach for sacropexy might cause difficulty of the surgical field in obese patients and that pectopexy could be appealing alternative option [10].

In comparison with sacropexy, pectopexy seems to have several benefits. First, the occurrence of intraoperative complications might be lower than sacropexy. The surgical fields of pectopexy contain lesser number of important structures. The preparation area of pectopexy contains external iliac vessels and obturator nerve and unlike sacropexy, does not include ureter or hypogastric vessels [10]. In fact, Noé et al. [11] reported the comparative study of laparoscopic pectopexy and laparoscopic sacral colpo-cervicopexy. In their study, the mean blood loss was significantly lesser in pectopexy group ( $4.6 \mathrm{~mL}$ vs. $15.3 \mathrm{~mL}, P<0.001$, respectively), and no intraoperative complications were reported with 43 pectopexy cases and 40 sacral colpopexy cases. In the prospective international, multicenter study published in 2020, the incidence of estimated blood loss (EBL) with more than $200 \mathrm{~mL}$ was $1.0 \%$ and that of organ damage was $0.8 \%$ during surgery or 14 days of postlaparoscopic pectopexy [12].

In our study, there were no intraoperative and postoperative complications. The mean EBL was $84 \mathrm{~mL}$. Twothirds of the patients underwent total hysterectomy or supracervical hysterectomy and thus the EBL was somewhat higher, compared with the cases of pectopexy only.

The other potential benefit of pectopexy is the lower incidence of gastrointestinal complications. Regarding sacrocolpopexy, the incidence of gastrointestinal complication was reported as more than 1\% [9]. Median incidence of postoperative ileus and small bowel obstruction were $3.6 \%$ and $1.1 \%$, respectively. In the study of Biler et al. [13], de novo persistent constipation was $7.1 \%$ in laparoscopic sacrocolpopexy and $0 \%$ in laparoscopic pectopexy $(P=0.19)$. However, no occurrence of constipation or bowel injury was reported in international study of pectopexy [12].

In the study of Noé et al. [11], they reported that the mean operating time was shorter in pectopexy group than sacrocolpopexy group. Yet, the operating time could be influenced by several factors such as experience of the operator, performance of hysterectomy and presence of concomitant surgeries.

Recently, Mairesse et al. [14] reported that concomitant hysterectomy in the first surgery was associated with a significantly lower risk of POP surgery recurrence (hazard ratio $[95 \%$ confidence interval] $=0.51$ [0.49-0.53]) [14]. Moreover, Dallas et al. [15] reported that hysterectomy at the time of prolapse repair was associated with a decreased risk of future POP surgery by $1 \%-3 \%$ in a large population-based cohort study. In their study, however, hysterectomy was independently associated with higher perioperative morbidity such as rates of blood transfusion, urologic injury or fistula.

In our study, we conducted mainly supracervical hysterectomy. In this case, dissection of vesico-vaginal space is not necessary, and associated complications such as bleeding and urologic injury might have been reduced. Also, because the mesh was fixed at cervix, the development of mesh-related complications such as inflammation and exposure of mesh could have been prevented. In this short-term follow-up study, there were no complications related with prosthetic mesh.

The current study has several limitations. First, only simple follow-up data, not comparative outcomes, was reported. For evaluating the benefits and/or risks of new surgical method, randomized controlled studies are inevitably required. Besides, the number of patients included in this study was relatively small, and the postoperative follow-up period was short. In spite of such limitations, this study is the first article reporting the clinical outcomes of pectopexy in Korean women. The 
outcomes of new surgical approach, pectopexy, seem promising and emanate the necessity of larger studies in near future.

\section{ACKNOWLEDGMENTS}

This work was supported by clinical research grant from Pusan National University Hospital in 2020.

\section{CONFLICT OF INTEREST}

No potential conflict of interest relevant to this article was reported.

\section{REFERENCES}

1. Barber MD, Maher C. Epidemiology and outcome assessment of pelvic organ prolapse. Int Urogynecol J 2013; 24: 1783-90.

2. American Urogynecologic Society. Pelvic organ prolapse. Female Pelvic Med Reconstr Surg 2019; 25: 397-408.

3. Yuk JS, Lee JH, Hur JY, Shin JH. The prevalence and treatment pattern of clinically diagnosed pelvic organ prolapse: a Korean National Health Insurance Database-based cross-sectional study 2009-2015. Sci Rep 2018; 8: 1334.

4. Dällenbach P. To mesh or not to mesh: a review of pelvic organ reconstructive surgery. Int J Womens Health 2015; 7: 331-43.

5. Giannini A, Russo E, Cano A, Chedraui P, Goulis DG, Lambrinoudaki I, et al. Current management of pelvic organ prolapse in aging women: EMAS clinical guide. Maturitas 2018; 110: 11823.

6. Madhu CK, Hashim H. Surgery for pelvic organ prolapse. Eur Urol Suppl 2018; 17: 119-25.

7. Nygaard IE, McCreery R, Brubaker L, Connolly A, Cundiff G, Weber AM, et al. Abdominal sacrocolpopexy: a comprehensive review. Obstet Gynecol 2004; 104: 805-23.

8. Sarlos D, Brandner S, Kots L, Gygax N, Schaer G. Laparoscopic sacrocolpopexy for uterine and post-hysterectomy prolapse: anatomical results, quality of life and perioperative outcome-a prospective study with 101 cases. Int Urogynecol J Pelvic Floor Dysfunct 2008; 19: 1415-22.

9. Whitehead WE, Bradley CS, Brown MB, Brubaker L, Gutman RE, Varner RE, et al. Gastrointestinal complications following abdominal sacrocolpopexy for advanced pelvic organ prolapse. Am J Obstet Gynecol 2007; 197: 78.e1-7.

10. Banerjee C, Noé KG. Laparoscopic pectopexy: a new technique of prolapse surgery for obese patients. Arch Gynecol Obstet 2011; 284: 631-5.

11. Noé KG, Spüntrup C, Anapolski M. Laparoscopic pectopexy: a randomised comparative clinical trial of standard laparoscopic sacral colpo-cervicopexy to the new laparoscopic pectopexy. Short-term postoperative results. Arch Gynecol Obstet 2013; 287: 275-80.

12. Noé GK, Schiermeier S, Papathemelis T, Fuellers U, Khudyakov A, Altmann $\mathrm{HH}$, et al. Prospective international multicenter pectopexy trial: Interim results and findings post surgery. Eur J Obstet Gynecol Reprod Biol 2020; 244: 81-6.

13. Biler A, Ertas IE, Tosun G, Hortu I, Turkay U, Gultekin OE, et al. Perioperative complications and short-term outcomes of abdominal sacrocolpopexy, laparoscopic sacrocolpopexy, and laparoscopic pectopexy for apical prolapse. Int Braz J Urol 2018; 44: 996-1004.

14. Mairesse S, Chazard E, Giraudet G, Cosson M, Bartolo S. Complications and reoperation after pelvic organ prolapse, impact of hysterectomy, surgical approach and surgeon experience. Int Urogynecol J 2020; 31: 1755-61.

15. Dallas K, Elliott CS, Syan R, Sohlberg E, Enemchukwu E, RogoGupta L. Association between concomitant hysterectomy and repeat surgery for pelvic organ prolapse repair in a cohort of nearly 100,000 women. Obstet Gynecol 2018; 132: 1328-36. 